\title{
Responding to complexity
}

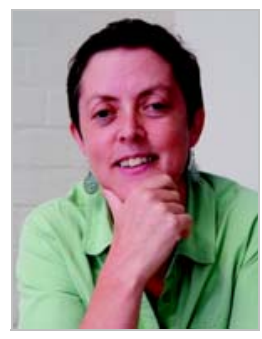

Diana McKay

Deputy Editor

mja@mja.com.au

doi: 10.5694/mjal4.c0407

admission rates for very old people between 1999 and

2011, a drop in rates for the 65-79-years age group meant there was an overall decrease in ICU admissions for older people. They also noted improved survival for older people admitted to the ICU. For the human face of the oldest old in the ICU, consider the recent experiences of a sprightly 90-year-old former editor of the New England Journal of Medicine - which are not contained in this issue but nevertheless well worth reading (http://www.nybooks.com/ articles/archives/2014/feb/06/on-breaking-ones-neck).

Schaefer and Brown (page 310) write of a distinctly different complexity: improving perinatal health care in resource-poor settings. With maternal and neonatal mortality rates starkly higher than those in Australia, they describe the initiatives of Médecins Sans Frontières in South Sudan. Key challenges include improving access to trained birth attendants, education for staff in neonatal care, and effective vaccination programs. Vaccination also remains topical in Australia. On a background of epidemic levels of pertussis in Queensland in 2009-2010, Sheridan and colleagues (page 334) confirmed the effectiveness of a primary course of acellular pertussis vaccine in protecting young children from pertussis notification and hospitalisation. However, their research accords with international findings that the vaccine's protection wanes in older children, compared with the previously used whole-cell vaccine. McIntyre and Nolan (page 306) comment that the acellular vaccine is undoubtedly effective at preventing severe pertussis but, given the evidence that its protection wanes over time, they consider other strategies that may further enhance Australia's immunisation response to pertussis.

Moving away from the bookends of life, Bennett (page 312) explores what the new federal government's priorities might be in its first term. She suggests a likely focus on efficiency, but notes this should support personcentred care in the correct setting, whether hospital or community-based, public or private. Also in the context of person-centred care, Ashby (page 305) discusses the role of innovative holistic approaches in treating persistent pain.

The Ethics series considers the fictional case of "John", a man with a history of mental illness and recent bereavement. His experience of care includes being transported to an emergency department by police. Bradbury and colleagues (page 348) consider why police transports for people under the New South Wales Mental Health Act 2007 have remained stable, despite legislative changes allowing trained ambulance staff to invoke the Act. They question whether a decisional capacity-based pathway would allow for earlier intervention, with the hope this would reduce the need for police involvement. Eagle and Ryan (page 352) report more broadly on patients who refuse treatment when they may be lacking capacity: a dilemma undoubtedly familiar to many readers. Without voice in this debate is John himself. Legislative changes may have a part to play in reducing coercive practice, but work in the field suggests that the involvement of people with a lived experience of mental illness is a key part of the complex interventions needed (Perm J 2011; 15: 57-62).

And, finally, fast food. Garcia and colleagues (page 340) explored the sodium content of Australian fast-food products between 2009 and 2012. While the mean sodium content per $100 \mathrm{~g}$ decreased, sodium levels in many products remained high, with wide variation between similar products suggesting further potential for reduction. Garcia et al argue for greater government engagement in food regulation to achieve more consistent sector-wide changes. A food labelling website, anyone?

\section{Health care + ageing $=$ jackpot}

Cate Swannell Careers Editor cswannell@mja.com.au
Private equity and venture capital have carved a small but significant niche in the finances of Australia's health care industry. How are these private funders influencing practice? Australia's ageing population, the increasing amount of money spent on health and our proximity to Asia have made our health care industry an attractive target for local and international investors. They are private equity groups with deep pockets and the ability to identify and amend operational inefficiencies and pursue opportunities for expansion.
Dr Mark Humphery-Jenner, senior lecturer in finance at the Australian School of Business at the University of New South Wales, says it's an ideal time for private equity involvement (page C1).

Social media use by doctors is in the spotlight with the release of new advertising guidelines by the Australian Health Practitioner Regulation Agency. What are the pitfalls (page C4)?

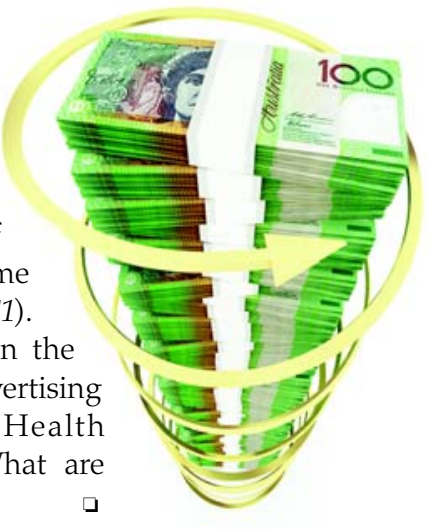

